\title{
Interactions Between the Soybean Cyst Nematode and Fusarium solani f. sp. glycines Based on Greenhouse Factorial Experiments
}

\author{
X. Gao, T. A. Jackson, G. L. Hartman, and T. L. Niblack
}

First and fourth authors: Department of Crop Sciences, University of Illinois Urbana 61801; second author: Department of Plant Pathology, University of Nebraska, Lincoln 68583; and third author: U.S. Department of Agriculture-Agricultural Research Service, 1101 W. Peabody, Urbana, IL 61801, and Department of Crop Sciences, University of Illinois, Urbana 61801. Accepted for publication 14 July 2006.

\begin{abstract}
Gao, X., Jackson, T. A., Hartman, G. L., and Niblack, T. L. 2006. Interactions between the soybean cyst nematode and Fusarium solani $\mathrm{f}$. sp. glycines based on greenhouse factorial experiments. Phytopathology 96:1409-1415.

The soybean cyst nematode, Heterodera glycines, and the fungus that causes sudden death syndrome (SDS) of soybean, Fusarium solani $\mathrm{f}$. sp. glycines, frequently co-infest soybean (Glycine max) fields. The interactions between $H$. glycines and $F$. solani $\mathrm{f}$. sp. glycines were investigated in

area under the SDS disease progress curve, and fungal colonization of roots. Both $H$. glycines and $F$. solani $\mathrm{f}$. sp. glycines reduced the growth of soybeans. Reproduction of $H$. glycines was suppressed by high inoculum levels but not by low levels of $F$. solani $\mathrm{f}$. sp. glycines. The infection of soybean roots by $H$. glycines did not affect root colonization by the fungus, as determined by real-time polymerase chain reaction. Although both pathogens reduced the growth of soybeans, $H$. glycines did not increase SDS foliar symptoms, and statistical interactions between the two pathogens were seldom significant.
\end{abstract} factorial greenhouse experiments with different inoculum levels of both organisms on a soybean cultivar susceptible to both pathogens. Measured responses included root and shoot dry weights, $H$. glycines reproduction,
Additional keywords: microbes, pathogen population ecology, quantitative plant pathology.
Both the soybean cyst nematode (Heterodera glycines) and Fusarium solani f. sp. glycines are major pathogens of soybean and can cause significant yield losses $(36,46)$. Both pathogens are soilborne and infect soybean roots. Although the total monetary loss due to $H$. glycines damage in the United States was estimated to be 1.1 billion dollars annually (36), the nematode does not always cause obvious aboveground symptoms (44). Under some conditions, soybeans infected by $F$. solani f. sp. glycines may develop soybean sudden death syndrome (SDS). This disease is characterized by root necrosis or rot, vascular discoloration of the roots and stem, and foliar chlorosis, necrosis, and premature defoliation $(16,29)$. The foliar symptoms of SDS are caused by phytotoxins produced by $F$. solani f. sp. glycines in the roots under favorable conditions for disease development $(13,14)$. F. solani $\mathrm{f}$. sp. glycines has been accepted as the causal organism for SDS, but recently the new name $F$. virguliforme Akoi, O'Donnell, Homma \& Lattanzi has been proposed to replace $F$. solani f. sp. glycines on the basis of morphological and phylogenetic studies (2). This new name has yet to be fully accepted (28); therefore, we refer to the SDS pathogen as F. solani f. sp. glycines in this paper.

The relationship between $H$. glycines and $F$. solani f. sp. glycines has been investigated for nearly 2 decades, but the interaction is not clearly understood. H. glycines is frequently present when SDS occurs (9). However, there are inconsistent reports concerning the effects of $H$. glycines on SDS. In greenhouse experiments, soybean seedlings inoculated simultaneously with $F$. solani f. sp. glycines and $H$. glycines developed more severe

Corresponding author: T. N. Niblack; E-mail address: tniblack@ uiuc.edu

DOI: 10.1094/PHYTO-96-1409

(C) 2006 The American Phytopathological Society foliar symptoms of SDS than soybean seedlings inoculated with the fungus alone $(18,21,23,30)$. In field experiments, symptoms of SDS increased when $H$. glycines was present, whereas $H$. glycines cyst numbers were suppressed by $F$. solani $\mathrm{f}$. sp. glycines $(21,33)$. Hershman et al. (8) reported, however, that there was no correlation between cyst population density and the area under SDS disease progress curve (AUDPC). Scherm et al. (35) reported a positive but weak relationship between $H$. glycines cyst counts and SDS symptoms in the field; their cross-correlation analysis indicated that $F$. solani f. sp. glycines densities and the number of $H$. glycines cysts were consistently associated with SDS severity. The interaction of $H$. glycines and SDS is not fully understood, as is indicated by both positive and negative or weak correlations of $H$. glycines population densities with the disease $(8,31,33,35)$.

Since the interaction between $H$. glycines and $F$. solani f. sp. glycines is still unclear, questions remain to be answered. For instance, does infestation level affect the interaction or will more sensitive detection methods, in particular real-time polymerase chain reaction (PCR) for quantification of $F$. solani f. sp. glycines (5), provide more insights into the interaction?

In this study, greenhouse experiments were used to (i) measure both the effects of $F$. solani f. sp. glycines infestation levels on $H$. glycines reproduction and final populations, and the effects of $H$. glycines infestation levels on $F$. solani f. sp. glycines colonization in root tissues as shown by quantification of fungal DNA, and (ii) compare the symptoms of host plants infected either singly or simultaneously with different levels of $H$. glycines and F. solani f. sp. glycines.

\section{MATERIALS AND METHODS}

H. glycines culture. An Hg-type 0 isolate of $H$. glycines designated PA3 (26) was maintained in a greenhouse at $27 \pm 1^{\circ} \mathrm{C}$ for 
30 days on soybean, Glycines max cv. Lee. H. glycines females and cysts were extracted from the roots and soil as previously described (27). Females and cysts were then placed on a 60-mesh sieve (250- $\mu \mathrm{m}$ aperture) and were manually ruptured with a rubber stopper to release the eggs, which were collected on a 500-mesh sieve (25- $\mu \mathrm{m}$ aperture) and then separated from debris by sucrose centrifugation (12). Eggs were rinsed into a 500-ml beaker, and sufficient tap water was added to dilute the suspension to 1,000 eggs per ml.

Fungal culture. Isolate Mont- 1 of $F$. solani f. sp. glycines was provided by the National Soybean Pathogen Collection Center at the University of Illinois and was maintained under constant fluorescent light at $24^{\circ} \mathrm{C}$ on water agar. To prepare inoculum, the fungus was grown on potato dextrose agar for 2 weeks and was then transferred to 1-liter flasks, each containing $200 \mathrm{~g}$ of red sorghum seed that had been soaked overnight and autoclaved twice. Each flask was infested with 10 plugs (8-mm-diameter) of the fungal culture and kept under constant fluorescent light at $24^{\circ} \mathrm{C}$. The inoculum in the flask was mixed by manual shaking every 2 to 3 days. After 2 weeks, the quantity of $F$. solani $\mathrm{f}$. sp. glycines DNA in the freeze-dried inoculum mixture, as determined by real-time PCR (5), was $310 \pm 152 \mathrm{pg} / \mathrm{mg}$.

Experimental design and conditions. All three experiments described in the following sections were set up in randomized complete blocks with four replications of each treatment. A silty clay loam soil (15\% sand, $49 \%$ silt, $36 \%$ clay [pH 6.1], and $4.2 \%$ organic matter) and a Topedo sand (93\% sand, 3\% silt, $4 \%$ clay [pH 8.5], and $0.2 \%$ organic matter) were collected from Champaign Country, Illinois. A steam-pasteurized soil mix (two parts sand to one part silty clay loam, giving a final mix of $77 \%$ sand, $11 \%$ silt, and $12 \%$ clay, with $\mathrm{pH} 7.8$, and $0.9 \%$ organic matter) was placed in $400-\mathrm{ml}$ tri-corner beakers $\left(350 \mathrm{~cm}^{3}\right.$ of soil mix per beaker) that were used as pots. All the $F$. solani f. sp. glycines inoculum was deposited in the middle of the pot as an evenly distributed layer. $H$. glycines eggs were pipetted into a hole $(8 \mathrm{~mm}$ wide by $30 \mathrm{~mm}$ deep). Seeds of soybean cv. Williams 82 were germinated for $48 \mathrm{~h}$ on damp germination paper, and one seedling was planted in each pot. The soil was maintained near waterholding capacity by watering once daily or as needed.

In one repetition (trial 1) of each experiment, pots were placed in a greenhouse water bath to maintain a constant root-zone temperature of $27 \pm 1^{\circ} \mathrm{C}$. The other repetition (trial 2) was conducted on a bench in one of two greenhouses where the air temperature ranged from 22.7 to $28.7^{\circ} \mathrm{C}$, with an average of $25.6^{\circ} \mathrm{C}$ during the experiments. The pots on the bench, as opposed to those in the water bath, were wrapped with aluminum foil to exclude light. Artificial light was provided for the greenhouse with the water bath (trial 1) at $14 \mathrm{~h} /$ day and for the other greenhouse (trial 2) at $16 \mathrm{~h} /$ day. The average supplemental light intensity was $21.5 \pm$ 3.0 klux in trial 1 and 9.2 \pm 2.6 klux in trial 2 .

Effect of inoculum level of $F$. solani $f$. sp. glycines (experiment 1). A $2 \times 3$ complete factorial design included all combinations of two levels of $H$. glycines ( 0 or 2,000 eggs per pot) and three levels of $F$. solani f. sp. glycines (none, low, or high). Three levels of $F$. solani f. sp. glycines inoculum were defined by the volume of inoculum added per pot. The "high" treatment received $15 \mathrm{~cm}^{3}$ of infested sorghum seed, whereas the "low" treatment received $1.5 \mathrm{~cm}^{3}$ of infested sorghum seed plus $13.5 \mathrm{~cm}^{3}$ of autoclaved infested sorghum seed. The "none" treatment received 15 $\mathrm{cm}^{3}$ of autoclaved infested sorghum seed.

Effect of $\boldsymbol{H}$. glycines inoculum level (experiment 2). A $4 \times 2$ complete factorial design included all combinations of four levels of $H$. glycines $(0,200,2,000$, or 20,000 eggs per pot) and two levels of $F$. solani f. sp. glycines (none or high); the "high" treatment received $15 \mathrm{~cm}^{3}$ of infested sorghum seed, whereas the "none" treatment received $15 \mathrm{~cm}^{3}$ of autoclaved infested sorghum seed.

Effect of high inoculum levels of $\boldsymbol{H}$. glycines and $\boldsymbol{F}$. solani $\mathrm{f}$. sp. glycines (experiment 3). A $2 \times 2$ complete factorial design included all combinations of two levels of $H$. glycines ( 0 or 20,000 eggs per pot) and two levels of $F$. solani $\mathrm{f}$. sp. glycines (none or high, i.e., $30 \mathrm{~cm}^{3}$ of autoclaved infested sorghum seed or $30 \mathrm{~cm}^{3}$ of infested sorghum seed per pot).

Data collection. Foliar symptoms were evaluated 12 days after planting and every 4 days thereafter with the following rating scale adapted from Hartman et al. (7): $0=$ no foliar symptoms; 1 = slight symptom development, with mottling and mosaic on leaves ( 1 to $20 \%$ of foliage affected); $2=$ moderate symptom development, with interveinal chlorosis and necrosis on foliage ( 21 to $50 \%$ of foliage affected); 3 = heavy symptom development, with interveinal chlorosis and necrosis (51 to $80 \%$ of foliage affected); and $4=$ severe interveinal chlorosis and necrosis (81 to $100 \%$ of foliage affected). This scale was used to calculate the area under the SDS disease progress curve (AUDPC) as described by Shaner and Finney (37).

The experiments were concluded after 6 weeks. The response variables evaluated included root necrosis, shoot height, flower number, root dry weight, plant dry weight, $H$. glycines eggs per pot, eggs per gram of dried root, and the AUDPC. H. glycines eggs were extracted as described previously (26) and stained with $0.035 \%$ acid fuchsin (10). Nematode reproduction factor (Rf) was calculated by dividing the final number of eggs per pot (Pf) by the initial number $(\mathrm{Pi}),(\mathrm{Pf}+1) /(\mathrm{Pi}+1)$. Root necrosis was visually rated as the proportion of necrosis in the whole root system, and flowers were counted. Plant shoots were dried at room temperature to less than $10 \%$ moisture, and the roots were freeze-dried for 3 days in a BenchTop 4K freeze dryer (VirTis, Gardiner, NY). After weighing, all of the freeze-dried roots from each replicate were ground in a cyclone sample mill (Model 3010, UDY Corp., Fort Collins, CO) (40), and the mill was cleaned manually between samples with high-pressure air. Ground roots were stored at $-20^{\circ} \mathrm{C}$.

Quantities of $\boldsymbol{F}$. solani f. sp. glycines DNA in roots. DNA was extracted as described in protocol 2 from Gao et al. (5) for quantification of $F$. solani f. sp. glycines in roots. Protocol 2 was adapted from Malvick and Grunden (19) and produced DNA extracts free of PCR inhibitors (5). Up to $50 \mathrm{mg}$ of freeze-dried root powder was used for DNA extraction. F. solani f. sp. glycines colonization levels in roots were determined with the real-time PCR protocol described previously (5).

Data analysis. Data were analyzed using version 8 of SAS (SAS Institute, Cary, NC). A two-way analysis of variance (ANOVA) model, the main statistical tool, was used to assess the effects of $H$. glycines, $F$. solani f. sp. glycines, and their interaction upon the growth of soybean. To assess the effect of the two trials in the greenhouse (with or without water bath control of soil temperature), a three-way ANOVA model was used; because the effect of trial was significant for most response variables other than root necrosis and AUDPC (data not shown), the data from trials 1 and 2 of each experiment were not combined. Egg numbers were transformed to $\log _{10}(x+1)$ values before analysis. Finally, the ANOVA for experiment 2 was supplemented with $t$ tests to determine whether $F$. solani f. sp. glycines was a significant factor at any of the four levels of nematode. A total of eight $t$ tests were conducted. The means were separated with Tukey's studentized range test at $\alpha=0.05$. To test whether the decline in $H$. glycines reproduction in $F$. solani f. sp. glycines-infested treatments resulted from detrimental effects of the fungus on the nematodes or from the reduction in available feeding sites as indicated by dry root weight, the number of $H$. glycines eggs per gram of dried root tissue was included as a parameter in the analysis.

Initial analysis of the DNA data was based on a mixed model in which the fixed effects were trial, $F$. solani $\mathrm{f}$. sp. glycines, and $H$. glycines. The random effect was the pot, which was nested within the particular combination of trial, F. solani f. sp. glycines treatment, and $H$. glycines treatment. Pot was included as a ran- 
dom effect because the data clearly indicated a significant pot effect. While this effect is not of primary interest, it must be included to achieve a correct analysis. The two-way and threeway interactions among trial, $F$. solani f. sp. glycines, and $H$. glycines were also included in the model. In cases where trial had a significant main effect or significant interactions with $F$. solani f. sp. glycines or $H$. glycines, an additional analysis was done separately for each of the two trials. The additional analysis employed a mixed model with $F$. solani f. sp. glycines and $H$. glycines as fixed effects, and pot as a random effect nested within $F$. solani f. sp. glycines $-H$. glycines combinations. In cases where trial had neither a significant main effect nor significant interactions with $F$. solani $\mathrm{f}$. sp. glycines or $H$. glycines, the data were merged across trials. In these cases, the additional analysis used a mixed model with $F$. solani $\mathrm{f}$. sp. glycines and $H$. glycines as fixed effects, and pot as a random effect within $F$. solani f. sp. glycines $-H$. glycines combinations.

\section{RESULTS}

Overall interaction between $\boldsymbol{H}$. glycines and $\boldsymbol{F}$. solani $\mathbf{f}$. sp. glycines. Statistical interactions between $H$. glycines and $F$. solani f. sp. glycines were seldom significant (Table 1), indicating that the effects of $H$. glycines and $F$. solani f. sp. glycines can be explained by an additive model. The strongest statistical effect in all three experiments was the main effect of $F$. solani f. sp. glycines, i.e., the fungus significantly reduced most response variables including $H$. glycines final population density (Tables 2,3 , and 4). The effect of the fungus on nematode population density was confirmed by $t$ tests with fixed $H$. glycines levels. These twosample $t$ tests showed significant differences in the numbers of eggs per pot according to $F$. solani f. sp. glycines inoculum level (data not shown).

Effect of $\boldsymbol{F}$. solani f. sp. glycines inoculum level (experiment 1). Results of experiment 1 , in which $H$. glycines was added at two levels ( 0 or 2,000 eggs per pot) and $F$. solani $\mathrm{f}$. sp. glycines at three levels $\left(0,1.5\right.$, or $15 \mathrm{~cm}^{3}$ of inoculum per pot), showed similar trends in both trials (Fig. 1; Table 2). SDS increased over time in all treatments containing $F$. solani f. sp. glycines except the treatment with the low level of the fungus in trial 1 (Fig. 1); no SDS developed in the treatments with autoclaved fungal inoculum. The AUDPC value with $F$. solani f. sp. glycines at high levels was statistically similar whether or not $H$. glycines was added, though it was highest when $H$. glycines was added (Table 2). Disease symptoms progressed more rapidly and the differences between treatments were more pronounced in trial 1 (constant

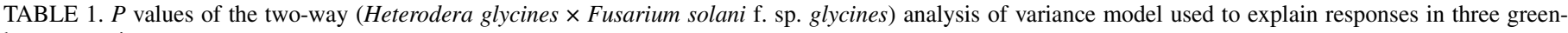
house experiments

\begin{tabular}{|c|c|c|c|c|c|c|}
\hline \multirow[b]{2}{*}{ Response variable } & \multicolumn{3}{|c|}{ Trial $1^{\mathrm{w}}$} & \multicolumn{3}{|c|}{ Trial $2^{\mathrm{x}}$} \\
\hline & Experiment 1 & Experiment 2 & Experiment 3 & Experiment 1 & Experiment 2 & Experiment 3 \\
\hline Root necrosis & 0.4233 & 0.0239 & 0.0006 & 0.4053 & 0.1104 & 0.2411 \\
\hline Plant height $(\mathrm{cm})$ & 0.2992 & 0.3511 & 0.0837 & 0.6084 & 0.4402 & 0.7365 \\
\hline Flowers/plant & 0.2155 & 0.8914 & 0.2149 & 0.4065 & 0.0041 & 0.1877 \\
\hline Root dry weight (g) & 0.0741 & 0.3979 & 0.2583 & 0.8748 & 0.4767 & 0.9904 \\
\hline Plant dry weight (g) & 0.5317 & 0.0642 & 0.1082 & 0.8890 & 0.0774 & 0.7633 \\
\hline Eggs/pot ${ }^{\mathrm{y}}$ & 0.0143 & 0.0020 & 0.0261 & 0.0230 & 0.0031 & 0.1950 \\
\hline AUDPC & 0.3665 & 0.0859 & 0.8465 & 0.0701 & 0.0357 & 0.4676 \\
\hline
\end{tabular}

${ }^{w}$ In trial 1, pots were in a water bath, which provided a constant root-zone temperature of $27 \pm 1^{\circ} \mathrm{C}$.

${ }^{x}$ In trial 2, pots sat on the greenhouse bench, and the average air temperature was $25.6^{\circ} \mathrm{C}\left(22.7\right.$ to $\left.28.7^{\circ} \mathrm{C}\right)$.

$\mathrm{y}$ The number of H. glycines eggs per pot was determined at the end of the experiment (6 weeks after planting).

${ }^{\mathrm{z}}$ Area under the SDS (soybean sudden death syndrome) disease progress curve.

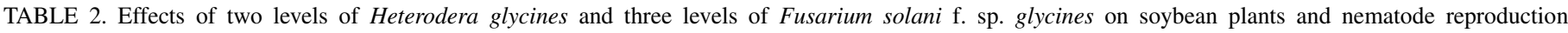
(experiment 1$)^{\mathrm{t}}$

\begin{tabular}{|c|c|c|c|c|c|c|c|c|c|c|}
\hline \multicolumn{2}{|c|}{ Treatment } & \multirow[b]{2}{*}{$\mathrm{AUDPC}^{\mathrm{v}}$} & \multirow[b]{2}{*}{$\begin{array}{c}\text { Root } \\
\text { necrosis }\end{array}$} & \multirow[b]{2}{*}{$\begin{array}{c}\text { Root dry } \\
\text { weight }(\mathrm{g})\end{array}$} & \multirow[b]{2}{*}{$\begin{array}{l}\text { Shoot dry } \\
\text { weight }(\mathrm{g})\end{array}$} & \multirow[b]{2}{*}{$\begin{array}{c}\text { Shoot } \\
\text { height }(\mathrm{cm})\end{array}$} & \multirow{2}{*}{$\begin{array}{c}\text { Whole } \\
\text { plant dry } \\
\text { weight }(\mathrm{g})\end{array}$} & \multirow[b]{2}{*}{$\begin{array}{c}\text { Flowers/ } \\
\text { plant }\end{array}$} & \multirow[b]{2}{*}{$\begin{array}{c}\text { Eggs/g of } \\
\text { root }\end{array}$} & \multirow[b]{2}{*}{$\begin{array}{l}\text { H. glycines } \\
\mathrm{Rf}^{\mathrm{x}}\end{array}$} \\
\hline $\begin{array}{l}\text { H. glycines } \\
\text { (eggs) }\end{array}$ & $\begin{array}{l}\text { F. solani f. sp. } \\
\text { glycines }^{\mathrm{u}}\end{array}$ & & & & & & & & & \\
\hline \multicolumn{11}{|l|}{ Trial $1^{\mathrm{y}}$} \\
\hline 2,000 & High & $80.4 \mathrm{a}$ & $0.88 \mathrm{a}$ & $0.12 \mathrm{~d}$ & $0.32 \mathrm{~d}$ & $9.9 \mathrm{~b}$ & $0.44 \mathrm{~d}$ & $1.3 \mathrm{a}$ & $36,109 \mathrm{a}$ & $3.85 \mathrm{~b}$ \\
\hline 0 & High & $50.5 \mathrm{ab}$ & $0.87 \mathrm{ab}$ & $0.28 \mathrm{c}$ & $0.66 \mathrm{~cd}$ & $12.5 \mathrm{ab}$ & $0.95 \mathrm{~cd}$ & $2.8 \mathrm{a}$ & 0 & 1.00 \\
\hline 2,000 & Low & $11.1 \mathrm{bc}$ & $0.86 \mathrm{ab}$ & $0.46 \mathrm{~b}$ & $0.99 \mathrm{bc}$ & $12.4 \mathrm{ab}$ & $1.45 \mathrm{bc}$ & $1.5 \mathrm{a}$ & $100,167 \mathrm{a}$ & $16.92 \mathrm{ab}$ \\
\hline 0 & Low & $0.0 \mathrm{c}$ & $0.74 \mathrm{ab}$ & $0.62 \mathrm{ab}$ & $1.49 \mathrm{ab}$ & $16.0 \mathrm{a}$ & $2.11 \mathrm{ab}$ & $1.5 \mathrm{a}$ & 0 & 1.00 \\
\hline 2,000 & 0 & $0.0 \mathrm{c}$ & $0.40 \mathrm{bc}$ & $0.70 \mathrm{a}$ & $1.54 \mathrm{ab}$ & $15.6 \mathrm{a}$ & $2.24 \mathrm{a}$ & $0.5 \mathrm{a}$ & $102,601 \mathrm{a}$ & $34.55 \mathrm{a}$ \\
\hline 0 & 0 & $0.0 \mathrm{c}$ & $0.11 \mathrm{c}$ & $0.62 \mathrm{ab}$ & $1.86 \mathrm{a}$ & $16.0 \mathrm{a}$ & $2.51 \mathrm{a}$ & $0.3 \mathrm{a}$ & 0 & 1.00 \\
\hline \multicolumn{11}{|l|}{ Trial $2^{\mathrm{z}}$} \\
\hline 2,000 & High & $53.1 \mathrm{a}$ & $0.79 \mathrm{a}$ & $0.77 \mathrm{~b}$ & $2.26 \mathrm{~b}$ & $20.3 \mathrm{a}$ & $3.03 \mathrm{~b}$ & $1.3 \mathrm{a}$ & $1,455 \mathrm{a}$ & $0.88 \mathrm{~b}$ \\
\hline 0 & High & $34.8 \mathrm{ab}$ & $0.85 \mathrm{a}$ & $0.86 \mathrm{~b}$ & $2.69 \mathrm{~b}$ & $22.2 \mathrm{a}$ & $3.66 \mathrm{~b}$ & $1.3 \mathrm{a}$ & 0 & 1.00 \\
\hline 2,000 & Low & $20.8 \mathrm{bc}$ & $0.65 \mathrm{a}$ & $1.11 \mathrm{~b}$ & $2.00 \mathrm{~b}$ & $20.8 \mathrm{a}$ & $3.11 \mathrm{~b}$ & $1.0 \mathrm{a}$ & 7,599 a & $4.68 \mathrm{ab}$ \\
\hline 0 & Low & $29.3 \mathrm{ab}$ & $0.66 \mathrm{a}$ & $1.09 \mathrm{~b}$ & $2.85 \mathrm{~b}$ & $19.2 \mathrm{a}$ & $3.93 \mathrm{~b}$ & $3.5 \mathrm{a}$ & 0 & 1.00 \\
\hline 2,000 & 0 & $0.0 \mathrm{c}$ & $0.25 \mathrm{~b}$ & $2.24 \mathrm{a}$ & $5.33 \mathrm{a}$ & $20.5 \mathrm{a}$ & $7.57 \mathrm{a}$ & $1.3 \mathrm{a}$ & $11,500 \mathrm{a}$ & $13.99 \mathrm{a}$ \\
\hline 0 & 0 & $0.0 \mathrm{c}$ & $0.17 \mathrm{~b}$ & $2.13 \mathrm{a}$ & $5.64 \mathrm{a}$ & $22.8 \mathrm{a}$ & $7.77 \mathrm{a}$ & $3.5 \mathrm{a}$ & 0 & 1.00 \\
\hline
\end{tabular}

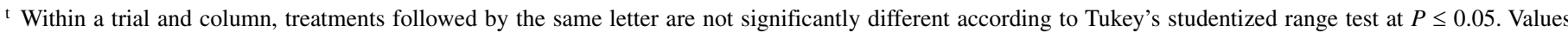
are the means of four replicate pots. For eggs per gram of root and $H$. glycines reproduction factor (Rf), data from pots not inoculated with $H$. glycines were excluded from the analysis.

"Inoculum consisted of colonized sorghum seeds.

${ }^{v}$ Area under the SDS (soybean sudden death syndrome) disease progress curve.

${ }^{\mathrm{w}}$ Root necrosis was visually rated as the proportion of necrosis in the whole root system.

${ }^{x} \mathrm{Rf}=($ final population +1$) /($ initial population +1$)$.

${ }^{y}$ In trial 1, pots were placed in a greenhouse water bath, which maintained a constant root-zone temperature of $27 \pm 1^{\circ} \mathrm{C}$.

${ }^{\mathrm{z}}$ In trial 2, pots sat on a bench in a greenhouse with an average air temperature of $25.6^{\circ} \mathrm{C}\left(22.7\right.$ to $\left.28.7^{\circ} \mathrm{C}\right)$. 
root-zone temperatures) than in trial 2. Presence of $H$. glycines with the low level of $F$. solani f. sp. glycines did not significantly affect SDS severity. Instead, SDS development was most dependent upon F. solani f. sp. glycines inoculum level (Fig. 1; Table 2).

$H$. glycines plus the high level of $F$. solani $\mathrm{f}$. sp. glycines reduced root dry weight more than either pathogen alone (Table 2). For the other variables, the combination of two pathogens had similar effects as single pathogen treatments. The high level of $F$. solani f. sp. glycines significantly reduced $H$. glycines reproduction, but the low level of the fungus did not (Table 2). Neither the high nor the low level of the fungus reduced the number of H. glycines eggs per gram of root (Table 2).
Effect of $\boldsymbol{H}$. glycines inoculum level (experiment 2). In treatments with $F$. solani f. sp. glycines, SDS increased over time in both trials (data not shown). AUDPC was not significantly greater in the presence of $H$. glycines and was independent of the three nematode levels (Table 3). Disease development was more rapid and the differences between symptom severities in the treatments were more pronounced in trial 1 (constant root-zone temperature) than in trial 2.

Dual infestation with a high level of $F$. solani f. sp. glycines and $20,000 \mathrm{H}$. glycines eggs caused severe root necrosis, but symptoms were not greater in this treatment than when 2,000 or 200 eggs were used with fungal inoculum in either trial (Table 3).

TABLE 3. Effects of four levels of Heterodera glycines and two levels of Fusarium solani f. sp. glycines on soybean plants and nematode reproduction (experiment 2$)^{\mathrm{t}}$

\begin{tabular}{|c|c|c|c|c|c|c|c|c|c|c|}
\hline \multicolumn{2}{|c|}{ Treatment } & \multirow[b]{2}{*}{$\mathrm{AUDPC}^{\mathrm{v}}$} & \multirow[b]{2}{*}{$\begin{array}{c}\text { Root } \\
\text { necrosis }\end{array}$} & \multirow[b]{2}{*}{$\begin{array}{c}\text { Root dry } \\
\text { weight }(\mathrm{g})\end{array}$} & \multirow[b]{2}{*}{$\begin{array}{l}\text { Shoot dry } \\
\text { weight }(\mathrm{g})\end{array}$} & \multirow[b]{2}{*}{$\begin{array}{c}\text { Shoot } \\
\text { height }(\mathrm{cm})\end{array}$} & \multirow{2}{*}{$\begin{array}{c}\text { Whole } \\
\text { plant dry } \\
\text { weight }(\mathrm{g})\end{array}$} & \multirow[b]{2}{*}{$\begin{array}{c}\text { Flowers/ } \\
\text { plant }\end{array}$} & \multirow[b]{2}{*}{$\begin{array}{l}\mathrm{Eggs} / \mathrm{g} \text { of } \\
\text { root }\end{array}$} & \multirow[b]{2}{*}{$\begin{array}{l}\text { H. glycines } \\
\operatorname{Rf}^{\mathrm{x}}\end{array}$} \\
\hline $\begin{array}{l}\text { H. glycines } \\
\text { (eggs) }\end{array}$ & $\begin{array}{l}\text { F. solani } \mathrm{f} . \mathrm{sp} \text {. } \\
\text { glycines }^{\mathrm{u}}\end{array}$ & & & & & & & & & \\
\hline \multicolumn{11}{|l|}{ Trial $1^{\mathrm{y}}$} \\
\hline 20,000 & High & $76.7 \mathrm{ab}$ & $0.96 \mathrm{a}$ & $0.09 \mathrm{c}$ & $0.18 \mathrm{c}$ & $9.4 \mathrm{~b}$ & $0.27 \mathrm{~d}$ & $1.3 \mathrm{a}$ & $1,089,892 \mathrm{a}$ & $1.56 \mathrm{~b}$ \\
\hline 2,000 & High & $88.5 \mathrm{a}$ & $0.83 \mathrm{a}$ & $0.11 \mathrm{c}$ & $0.18 \mathrm{~d}$ & $11.2 \mathrm{~b}$ & $0.29 \mathrm{~d}$ & $1.0 \mathrm{a}$ & $135,600 \mathrm{a}$ & $4.66 \mathrm{~b}$ \\
\hline 200 & High & $49.3 \mathrm{ab}$ & $0.85 \mathrm{a}$ & $0.13 \mathrm{bc}$ & $0.37 \mathrm{~cd}$ & $10.3 \mathrm{~b}$ & $0.50 \mathrm{~d}$ & $2.7 \mathrm{a}$ & $9,951 \mathrm{~b}$ & $5.99 \mathrm{~b}$ \\
\hline 0 & High & $38.8 \mathrm{abc}$ & $0.68 \mathrm{ab}$ & $0.09 \mathrm{c}$ & $0.28 \mathrm{bc}$ & $10.2 \mathrm{~b}$ & $0.37 \mathrm{~d}$ & $1.3 \mathrm{a}$ & 0 & 1.00 \\
\hline 20,000 & 0 & $0 \mathrm{c}$ & $0.82 \mathrm{a}$ & $0.36 \mathrm{ab}$ & $0.76 \mathrm{ab}$ & $12.8 \mathrm{ab}$ & $1.12 \mathrm{bc}$ & $1.3 \mathrm{a}$ & $438,641 \mathrm{a}$ & $6.81 \mathrm{~b}$ \\
\hline 2,000 & 0 & $0 \mathrm{c}$ & $0.35 \mathrm{bc}$ & $0.55 \mathrm{a}$ & $1.26 \mathrm{ab}$ & $16.3 \mathrm{a}$ & $1.81 \mathrm{ab}$ & $2.8 \mathrm{a}$ & $102,175 \mathrm{a}$ & $28.20 \mathrm{a}$ \\
\hline 200 & 0 & $0 \mathrm{c}$ & $0.08 \mathrm{c}$ & $0.59 \mathrm{a}$ & $1.56 \mathrm{a}$ & $17.5 \mathrm{a}$ & $2.15 \mathrm{a}$ & $3.0 \mathrm{a}$ & $8,931 \mathrm{~b}$ & $25.30 \mathrm{a}$ \\
\hline 0 & 0 & $0 \mathrm{c}$ & $0.08 \mathrm{c}$ & $0.40 \mathrm{ab}$ & $1.58 \mathrm{a}$ & $16.6 \mathrm{a}$ & $1.98 \mathrm{a}$ & $2.8 \mathrm{a}$ & 0 & 1.00 \\
\hline \multicolumn{11}{|l|}{ Trial $2^{\mathrm{z}}$} \\
\hline 20,000 & High & $55.7 \mathrm{a}$ & $0.96 \mathrm{a}$ & $0.65 \mathrm{~b}$ & $1.52 \mathrm{c}$ & $16.0 \mathrm{a}$ & $2.17 \mathrm{c}$ & $0 \mathrm{~b}$ & $24,562 \mathrm{ab}$ & $0.81 \mathrm{c}$ \\
\hline 2,000 & High & $49.7 \mathrm{a}$ & $0.93 \mathrm{ab}$ & $0.76 \mathrm{~b}$ & $2.08 \mathrm{bc}$ & $21.3 \mathrm{a}$ & $2.84 \mathrm{c}$ & $2.5 \mathrm{a}$ & $3,501 \mathrm{ab}$ & $1.32 \mathrm{c}$ \\
\hline 200 & High & $45.4 \mathrm{a}$ & $0.91 \mathrm{ab}$ & $0.72 \mathrm{~b}$ & $1.77 \mathrm{c}$ & $19.4 \mathrm{a}$ & $2.49 \mathrm{c}$ & $0.3 \mathrm{~b}$ & $34 \mathrm{c}$ & $0.14 \mathrm{c}$ \\
\hline 0 & High & $38.3 \mathrm{a}$ & $0.89 a b$ & $0.72 \mathrm{~b}$ & $1.89 \mathrm{c}$ & $17.6 \mathrm{a}$ & $2.60 \mathrm{c}$ & $0.3 \mathrm{~b}$ & 0 & 1.00 \\
\hline 20,000 & 0 & $0 \mathrm{~b}$ & $0.56 \mathrm{bc}$ & $1.87 \mathrm{a}$ & $3.27 \mathrm{~b}$ & $18.3 \mathrm{a}$ & $5.14 \mathrm{~b}$ & $0 \mathrm{~b}$ & $73,948 \mathrm{a}$ & $7.45 \mathrm{~b}$ \\
\hline 2,000 & 0 & $0 \mathrm{~b}$ & $0.41 \mathrm{dc}$ & $2.12 \mathrm{a}$ & $4.66 \mathrm{a}$ & $21.0 \mathrm{a}$ & $6.78 \mathrm{~b}$ & $0 \mathrm{~b}$ & $17,288 \mathrm{ab}$ & $18.02 \mathrm{a}$ \\
\hline 200 & 0 & $0 \mathrm{~b}$ & $0.13 \mathrm{~d}$ & $2.35 \mathrm{a}$ & $5.04 \mathrm{a}$ & $22.3 \mathrm{a}$ & $7.38 \mathrm{a}$ & $0.3 \mathrm{~b}$ & $779 \mathrm{~b}$ & $9.29 \mathrm{ab}$ \\
\hline 0 & 0 & $0 \mathrm{~b}$ & $0.19 \mathrm{dc}$ & $2.14 \mathrm{a}$ & $4.96 \mathrm{a}$ & $22.0 \mathrm{a}$ & $7.10 \mathrm{a}$ & $0.3 \mathrm{~b}$ & 0 & 1.00 \\
\hline
\end{tabular}

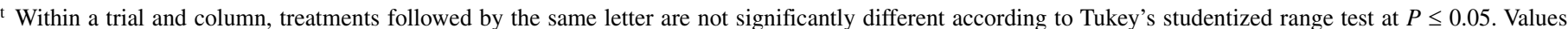
are the means of four replicate pots. For eggs per gram of root and $H$. glycines reproduction factor (Rf), data from pots not inoculated with $H$. glycines were excluded from the analysis.

" Inoculum consisted of colonized sorghum seeds.

v Area under the SDS (soybean sudden death syndrome) disease progress curve.

${ }^{w}$ Root necrosis was visually rated as the proportion of necrosis in the whole root system.

${ }^{\mathrm{x}} \mathrm{Rf}=($ final population +1$) /($ initial population +1$)$.

y In trial 1, pots were placed in a greenhouse water bath, which maintained a constant root-zone temperature of $27 \pm 1^{\circ} \mathrm{C}$

${ }^{\mathrm{z}}$ In trial 2, pots sat on a bench in a greenhouse with an average air temperature of $25.6^{\circ} \mathrm{C}\left(22.7\right.$ to $\left.28.7^{\circ} \mathrm{C}\right)$.

TABLE 4. Effects of high inoculum levels of Heterodera glycines and Fusarium solani f. sp. glycines on soybean plants and nematode reproduction (experiment 3$)^{\mathrm{t}}$

\begin{tabular}{|c|c|c|c|c|c|c|c|c|c|c|}
\hline \multicolumn{2}{|c|}{ Treatment } & \multirow[b]{2}{*}{ AUDPC $^{\mathrm{v}}$} & \multirow[b]{2}{*}{$\begin{array}{c}\text { Root } \\
\text { necrosis }\end{array}$} & \multirow[b]{2}{*}{$\begin{array}{c}\text { Root dry } \\
\text { weight (g) }\end{array}$} & \multirow[b]{2}{*}{$\begin{array}{l}\text { Shoot dry } \\
\text { weight }(\mathrm{g})\end{array}$} & \multirow[b]{2}{*}{$\begin{array}{c}\text { Shoot } \\
\text { height }(\mathrm{cm})\end{array}$} & \multirow{2}{*}{$\begin{array}{c}\text { Whole } \\
\text { plant dry } \\
\text { weight }(\mathrm{g})\end{array}$} & \multirow[b]{2}{*}{$\begin{array}{c}\text { Flowers/ } \\
\text { plant }\end{array}$} & \multirow[b]{2}{*}{$\begin{array}{l}\mathrm{Eggs} / \mathrm{g} \text { of } \\
\text { root }\end{array}$} & \multirow[b]{2}{*}{$\begin{array}{l}\text { H. glycines } \\
\text { Rf }^{\mathrm{x}}\end{array}$} \\
\hline $\begin{array}{l}\text { H. glycines } \\
\text { (eggs) }\end{array}$ & $\begin{array}{l}\text { F. solani } \mathrm{f} . \mathrm{sp} \text {. } \\
\text { glycines }^{\mathrm{u}}\end{array}$ & & & & & & & & & \\
\hline \multicolumn{11}{|l|}{ Trial $1^{\mathrm{y}}$} \\
\hline 20,000 & Very high & $100.8 \mathrm{a}$ & $0.95 \mathrm{a}$ & $0.03 \mathrm{~b}$ & $0.08 \mathrm{~b}$ & $8.8 \mathrm{~b}$ & $0.10 \mathrm{a}$ & $0 \mathrm{~b}$ & $9,500 \mathrm{a}$ & $0.02 \mathrm{~b}$ \\
\hline 0 & Very high & $96.9 \mathrm{a}$ & $0.96 \mathrm{a}$ & $0.05 \mathrm{~b}$ & $0.09 \mathrm{~b}$ & $7.3 \mathrm{~b}$ & $0.13 \mathrm{a}$ & $0 \mathrm{~b}$ & 0 & 1.00 \\
\hline 20,000 & 0 & $0.0 \mathrm{c}$ & $0.66 \mathrm{~b}$ & $0.33 \mathrm{a}$ & $0.81 \mathrm{a}$ & $16.3 \mathrm{a}$ & $1.13 \mathrm{~b}$ & $2.5 \mathrm{ab}$ & $7,061 \mathrm{a}$ & $0.13 \mathrm{a}$ \\
\hline 0 & 0 & $0.0 \mathrm{c}$ & $0.13 \mathrm{c}$ & $0.44 \mathrm{a}$ & $1.28 \mathrm{a}$ & $12.9 \mathrm{a}$ & $1.72 \mathrm{~b}$ & $4.5 \mathrm{a}$ & 0 & 1.00 \\
\hline \multicolumn{11}{|l|}{ Trial $2^{\mathrm{z}}$} \\
\hline 20,000 & Very high & $48.6 \mathrm{a}$ & $0.95 \mathrm{a}$ & $0.37 \mathrm{~b}$ & $0.84 \mathrm{~b}$ & $15.9 \mathrm{~b}$ & $1.20 \mathrm{~b}$ & $0 \mathrm{a}$ & $378 \mathrm{a}$ & $0.01 \mathrm{a}$ \\
\hline 0 & Very high & $38.7 \mathrm{a}$ & $0.92 \mathrm{a}$ & $0.41 \mathrm{~b}$ & $1.00 \mathrm{~b}$ & $16.0 \mathrm{~b}$ & $1.40 \mathrm{~b}$ & $0 \mathrm{a}$ & 0 & 1.00 \\
\hline 20,000 & 0 & $1.5 \mathrm{c}$ & $0.59 \mathrm{ab}$ & $1.23 \mathrm{ab}$ & $3.13 \mathrm{a}$ & $21.4 \mathrm{a}$ & $4.36 \mathrm{a}$ & $0.5 \mathrm{a}$ & $26,005 \mathrm{a}$ & $1.92 \mathrm{a}$ \\
\hline 0 & 0 & $0.6 \mathrm{c}$ & $0.32 \mathrm{~b}$ & $1.28 \mathrm{a}$ & $3.62 \mathrm{a}$ & $22.5 \mathrm{a}$ & $4.89 \mathrm{a}$ & $0 \mathrm{a}$ & 0 & 1.00 \\
\hline
\end{tabular}

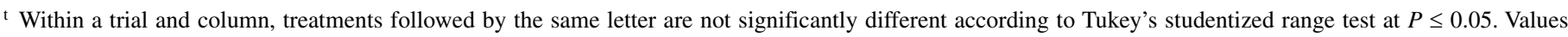
are the means of four replicate pots. For eggs per gram of root and $H$. glycines reproduction factor (Rf), data from pots not inoculated with $H$. glycines were excluded from the analysis.

"Inoculum consisted of colonized sorghum seeds.

${ }^{v}$ Area under the SDS (soybean sudden death syndrome) disease progress curve.

${ }^{\mathrm{w}}$ Root necrosis was visually rated as the proportion of necrosis in the whole root system.

${ }^{x} \mathrm{Rf}=($ final population +1$) /($ initial population +1$)$.

${ }^{y}$ In trial 1, pots were placed in a greenhouse water bath, which maintained a constant root-zone temperature of $27 \pm 1^{\circ} \mathrm{C}$.

${ }^{\mathrm{z}}$ In trial 2, pots sat on a bench in a greenhouse with an average air temperature of $25.6^{\circ} \mathrm{C}\left(22.7\right.$ to $\left.28.7^{\circ} \mathrm{C}\right)$. 
Root and shoot dry weights were reduced in the treatments containing high levels of $F$. solani f. sp. glycines. Nematode reproduction was reduced by the fungus and by the high initial level of nematode infestation. Plant dry weight was reduced and root necrosis was increased by increasing $H$. glycines population densities, although not always significantly.

Nematode reproduction was significantly greater in trial 1 (constant root-zone temperature) than in trial 2 (Table 3). Nematode reproduction only occurred in $H$. glycines-infested treatments and was reduced by $F$. solani $\mathrm{f}$. sp. glycines inoculum regardless of nematode inoculum level. As in experiment 1 , however, $F$. solani f. sp. glycines did not affect numbers of nematode eggs per gram of root (Table 3 ). Thus, the fungus reduced nematode reproduction by reducing root mass.

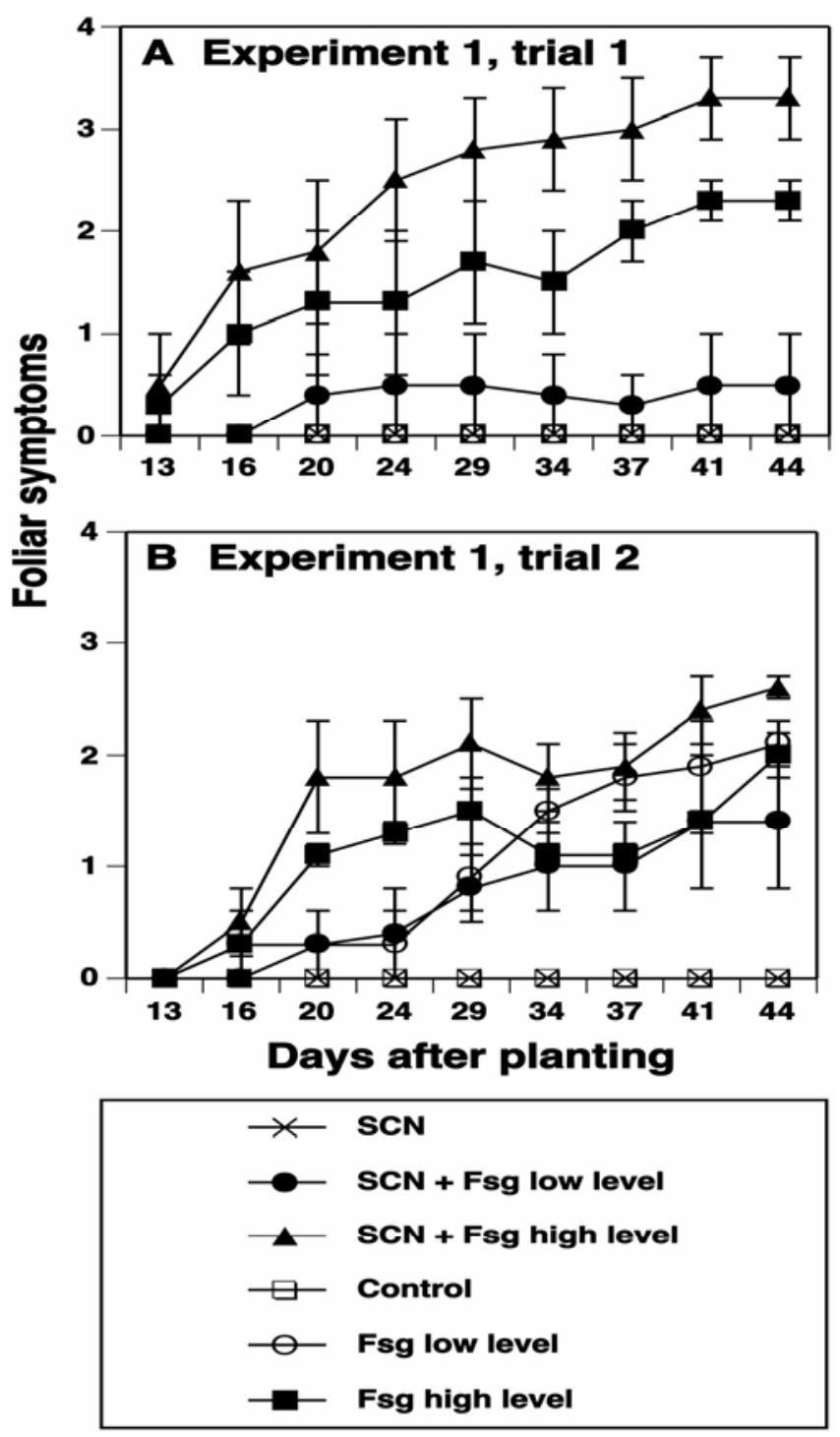

Fig. 1. Development of foliar symptoms of soybean sudden death syndrome on soybean plants infested with three levels of Fusarium solani f. sp. glycines (Fsg) and two levels of Heterodera glycines (SCN) in experiment 1: A, trial 1, with a constant root-zone temperature of $27 \pm 1{ }^{\circ} \mathrm{C}$; and $\mathbf{B}$, trial 2, with an average air temperature of $25.6^{\circ} \mathrm{C}\left(22.7\right.$ to $\left.28.7^{\circ} \mathrm{C}\right)$. Foliar symptoms were rated with the following scale: $0=$ no foliar symptoms; $1=$ slight symptom development, with mottling and mosaic on leaves (1 to $20 \%$ of foliage affected); $2=$ moderate symptom development, with interveinal chlorosis and necrosis ( 21 to $50 \%$ of foliage affected); $3=$ heavy symptom development, with interveinal chlorosis and necrosis (51 to $80 \%$ of foliage affected); and $4=$ severe interveinal chlorosis and necrosis ( 81 to $100 \%$ of foliage affected). Values are the means of four replications. For trial 1, there were no foliar symptoms with the noninfested control, SCN alone, or Fsg low level alone. For trial 2, there were no foliar symptoms with the noninfested control or SCN alone.
Effect of high levels of $\boldsymbol{H}$. glycines and $\boldsymbol{F}$. solani f. sp. glycines (experiment 3). In $F$. solani $\mathrm{f}$. sp. glycines-infested treatments, AUDPC was not significantly affected by $H$. glycines in either trial (Table 4). Some foliar necrosis was observed in the two treatments lacking $F$. solani f. sp. glycines in trial 2 , and although the cause was unknown, the mean AUDPC was significantly less than in the $F$. solani f. sp. glycines-infested treatments. SDS development was less severe in trial 2 than in trial 1 (Table 4).

When added separately, the fungus increased root necrosis (Table 4). In addition, root, shoot, and whole plant dry weights were reduced by $F$. solani f. sp. glycines (Table 4). Plants did not flower in the $F$. solani f. sp. glycines-infested treatments. Nematode reproduction was similar in trial 1 and trial 2 of experiment 3 but was less than in experiments 1 and 2. H. glycines reproduction was reduced by $F$. solani f. sp. glycines in trial 1 but not in trial 2 .

Quantities of $F$. solani f. sp. glycines DNA in roots. To analyze the DNA data for the three experiments, both nested and averaged models were initially considered. Because it maintained the full structure of the data set and did not require data averaging, the nested model was selected.

In experiment 1 , trial was not a significant main effect for either $F$. solani f. sp. glycines or $H$. glycines, and therefore the data from the two trials were merged. In the analysis, the only significant main effect was that of $F$. solani $\mathrm{f}$. sp. glycines level; neither $H$. glycines nor its interaction with $F$. solani f. sp. glycines was significant (Table 5). F. solani $\mathrm{f}$. sp. glycines DNA was unexpectedly detected in roots of noninoculated control plants but an additional ANOVA indicated that the quantity of DNA was less $(P=0.0214)$ in control plants than in plants inoculated with F. solani f. sp. glycines.

TABLE 5. Quantities of Fusarium solani f. sp. glycines DNA in soybean roots as determined by real-time polymerase chain reaction in experiment 1

\begin{tabular}{|c|c|c|c|}
\hline \multicolumn{2}{|c|}{ Treatment } & \multirow{2}{*}{$\begin{array}{l}\text { F. solani f. sp. } \\
\text { glycines DNA } \\
(\mathrm{pg} / \mathrm{mg} \text { of root })^{\mathrm{z}}\end{array}$} & \multirow[b]{2}{*}{ Standard erro } \\
\hline $\begin{array}{l}\text { Heterodera } \\
\text { glycines (eggs) }\end{array}$ & $\begin{array}{l}\text { F. solani } \mathrm{f} . \mathrm{sp} . \\
\text { glycines }^{\mathrm{y}}\end{array}$ & & \\
\hline 2,000 & High & $16,100 \mathrm{ab}$ & 9,088 \\
\hline 0 & High & $23,784 \mathrm{ab}$ & 7,676 \\
\hline 2,000 & Low & $35,236 \mathrm{~b}$ & 7,181 \\
\hline 0 & Low & $24,701 \mathrm{ab}$ & 7,181 \\
\hline 2,000 & 0 & $11,786 \mathrm{a}$ & 7,183 \\
\hline 0 & 0 & $5,717 \mathrm{a}$ & 8,291 \\
\hline
\end{tabular}

${ }^{\mathrm{y}}$ Inoculum consisted of colonized sorghum seeds.

${ }^{\mathrm{z}}$ Least squares means of DNA quantity of $F$. solani $\mathrm{f}$. sp. glycines in soybean roots. For each column, means followed by the same letter are not significantly different according to the Tukey pairwise comparison at $\alpha=0.05$ in mixed procedure. Values are the means of 24 replications (trials 1 and 2 combined).

TABLE 6. Quantities of Fusarium solani f. sp. glycines DNA in soybean roots as determined by real-time polymerase chain reaction in experiment 2

\begin{tabular}{|c|c|c|c|c|c|}
\hline \multirow{2}{*}{\multicolumn{2}{|c|}{ Treatment }} & \multicolumn{4}{|c|}{ F. solani f. sp. glycines DNA (pg/mg of root $)^{2}$} \\
\hline & & \multicolumn{2}{|c|}{ Trial 1} & \multicolumn{2}{|c|}{ Trial 2} \\
\hline $\begin{array}{l}\text { Heterodera } \\
\text { glycines (eggs) }\end{array}$ & $\begin{array}{l}\text { F. solani } \mathrm{f} \text {. } \\
\text { sp. glycines }\end{array}$ & Mean & $\begin{array}{l}\text { Standard } \\
\text { error }\end{array}$ & Mean & $\begin{array}{c}\text { Standard } \\
\text { error }\end{array}$ \\
\hline 20,000 & High & $32,903 \mathrm{~b}$ & 3,601 & $20,532 \mathrm{~b}$ & 5,420 \\
\hline 2,000 & High & $16,910 \mathrm{a}$ & 5,092 & $25,721 \mathrm{bc}$ & 5,420 \\
\hline 200 & High & $13,000 \mathrm{a}$ & 5,092 & $44,483 \mathrm{~d}$ & 5,420 \\
\hline 0 & High & $16,761 \mathrm{a}$ & 5,092 & $37,334 \mathrm{~cd}$ & 5,420 \\
\hline 20,000 & 0 & $7,418 \mathrm{a}$ & 5,092 & $2,260 \mathrm{a}$ & 5,420 \\
\hline 2,000 & 0 & $6,665 \mathrm{a}$ & 3,601 & $3,672 \mathrm{a}$ & 5,420 \\
\hline 200 & 0 & $5,043 \mathrm{a}$ & 3,601 & $910 \mathrm{a}$ & 6,258 \\
\hline 0 & 0 & $4,606 \mathrm{a}$ & 3,601 & $1,704 \mathrm{a}$ & 6,258 \\
\hline
\end{tabular}

${ }^{\mathrm{z}}$ Least squares means of DNA quantity of $F$. solani f. sp. glycines in soybean roots. Within each trial and column, means followed by the same letter are not significantly different according to the Tukey pairwise comparison $\alpha=$ 0.05 in mixed procedure. Values are the means of 12 replications. 
In experiment 2, trial had significant two-way and three-way interactions with $F$. solani f. sp. glycines and $H$. glycines, and therefore separate analyses were conducted for each trial. As in experiment 1 , the only significant main effect was that of $F$. solani f. sp. glycines, and neither $H$. glycines nor its interaction with F. solani f. sp. glycines was significant (Table 6). Again, F. solani f. sp. glycines DNA was detected in the noninoculated controls but the quantity was less $(P=0.0004$ in trial 1 and 0.0001 in trial 2 ) in control plants than in the inoculated plants.

In experiment 3, it was only possible to run the initial model with main effects and two-way interactions because of missing data. Because the main effect of trial was significant, two additional analyses were done: the data from the two trials were merged or analyzed separately. In both analyses, neither of the main effects for $F$. solani $\mathrm{f}$. sp. glycines and $H$. glycines nor their interactions were significant (data not shown). DNA was again detected in the noninoculated controls but there were no significant differences in DNA quantities $(P=0.3184$ in trial 1 and 0.6219 in trial 2) in control and inoculated plants, probably due to the severe root necrosis in the inoculated plants (Table 4).

\section{DISCUSSION}

Plant-parasitic nematodes in the rhizosphere co-exist and may interact with numerous microbes and other organisms (15). Those nematodes, like $H$. glycines, that enter roots are also likely to interact with plant-pathogenic fungi and bacteria within the roots of common host plants $(3,15,34,43)$. Because $H$. glycines and $F$. solani f. sp. glycines are common and important soilborne pathogens of soybean, understanding how the plant responds to combinations of these pathogens is important. Contrary to previous reports $(18,21,30,32)$, our greenhouse studies indicated that $H$. glycines did not increase severity of foliar symptoms of SDS caused by $F$. solani f. sp. glycines. This may be due to the use of different isolates and inoculum levels of $H$. glycines and $F$. solani f. sp. glycines, and/or different varieties of soybean. A number of reports have indicated that the nematode populations, pathogenic fungal isolates, plant cultivars, and experimental conditions can affect the interactions between nematodes and other plant pathogens $(20,38,45)$.

Sikora and Carter (39) emphasized that a range of inoculum densities of both pathogens should be used in various environmental conditions to determine the overall influence of normal and extreme conditions on the interaction. For example, inoculum densities affected the interactions between Meloidogyne incognita and $F$. oxysporum f. sp. vasinfectum on cotton $(6,41)$. Previous studies of interactions between $H$. glycines and $F$. solani f. sp. glycines have not included different inoculum levels. We found that $F$. solani f. sp. glycines suppressed $H$. glycines egg populations only when the $F$. solani $\mathrm{f}$. sp. glycines inoculum was high, indicating that fungal population level plays an important role in the interaction with the nematode. Because the fungus did not reduce the number of eggs produced per gram of dried root, the reduced $H$. glycines reproduction per plant probably resulted from the reduction in root material available for nematode feeding.

Understanding how nematodes and fungal pathogens interact depends on advances in research methods and tools. Although $H$. glycines can be conveniently enumerated with a conventional microscope, $F$. solani f. sp. glycines cannot. Use of semiselective media to enumerate the colony forming units of Fusarium spp. glycines in roots $(4,11,25)$ is time-consuming and labor intensive. We used a real-time PCR assay for quantification of $F$. solani $\mathrm{f}$. sp. glycines and found that the infection of soybean roots by $H$. glycines did not significantly affect the colonization of plant tissues by $F$. solani f. sp. glycines.

The use of real-time PCR to quantify a plant pathogen's DNA was very useful but there is limited information available on how to statistically analyze these kinds of data. In our statistical analy- sis of $F$. solani f. sp. glycines DNA quantities, we selected a nested model after examining both nested and averaged models. The nested model was considered more appropriate and powerful than the averaged one because it captured the full structure of the data and did not sacrifice information, i.e., it did not require averaging of the individual data points.

Small quantities of $F$. solani f. sp. glycines DNA were detected in some of our control plants. Such fungal contamination is not uncommon in greenhouse experiments and usually results from experimental manipulation or greenhouse insects $(1,17,24,42)$. Although sometimes contaminated with $F$. solani f. sp. glycines, the control plants in our study remained useful as controls because they contained much less fungal DNA than treated plants, at least when the inoculum levels were not extremely high, and were usually symptom free.

In summary, two major conclusions can be drawn from our greenhouse experiments. First, the effect of $H$. glycines on $F$. solani f. sp. glycines root colonization was not significant. Second, $H$. glycines did not increase SDS symptom severity under our experimental conditions. Further studies on the interaction between $H$. glycines and SDS should include a wider range of F. solani f. sp. glycines inoculum levels at planting.

\section{ACKNOWLEDGMENTS}

We thank A. Martinsek (director of Illinois Statistical Office [ISO]), Y. Qi, H. Feng, B. Gao, E. Xu, J. Ling, P. P. Chow, and S. Fang for excellent statistical consultation and assistance; D. Malvick and E. Grunden for their fungal DNA extraction method from plant tissues; S. Li and the Soybean Pathogen Germplasm Collection for fungal culture assistance; G. Sims and L. Connor for help in freeze drying root samples; K. Colgrove, M. Raymond, and U. Reuter-Carlson for technical assistance; K. Lambert for valuable discussions; and A. Colgrove, S. Bekal, and two anonymous reviewers for critically reading the manuscript. This project was supported by the Illinois Soybean Program Operating Board.

\section{LITERATURE CITED}

1. Abawi, G. S., and Barker, K. R. 1983. Effects of cultivar, soil temperature, and population levels of Meloidogyne incognita on root necrosis and Fusarium wilt of tomato. Phytopathology 74:433-438.

2. Akoi, T., O’Donnell, K., Homma, Y., and Lattanzi, A. R. 2003. Sudden death syndrome of soybean is caused by two morphologically and phylogenetically distinct species within the $F$. solani species complex $-F$. virguliforme in North America and F. tucumaniae in South America. Mycologia 95:660-680.

3. Bond, J., and Wrather, J. A. 2004. Interaction with other plant pathogens and pests. Pages 111-129 in: Biology and Management of Soybean Cyst Nematode. 2nd ed. D. P. Schmitt, J. A. Wrather, and R. D. Riggs, eds. Schmitt \& Associates, Marceline, MO

4. Cho, J. H., Rupe, J. C., Cummings, M. S., and Gbur, E. E., Jr. 2001. Isolation and identification of Fusarium solani f. sp. glycines from soil on modified Nash and Snider's medium. Plant Dis. 85:256-260.

5. Gao, X., Jackson, T. A., Lambert, K. N., Li, S., Hartman, G. L., and Niblack, T. L. 2004. Detection and quantification of Fusarium solani $\mathrm{f}$. sp. glycines in soybean roots using real-time quantitative polymerase chain reaction. Plant Dis. 88:1372-1380

6. Garber, R. H., Jorgenson, E. C., Smith, S., and Hyer, A. H. 1979. Interaction of population levels of Fusarium oxysporum f. sp. vasinfectum and Meloidogyne incognita on cotton. J. Nematol. 11:133-137.

7. Hartman, G. L., Huang, Y. H., Nelson, R. L., and Noel, G. R. 1997. Germplasm evaluation of Glycine max for resistance to Fusarium solani, the causal agent of sudden death syndrome of soybean. Plant Dis. 81:515518 .

8. Hershman, D. E., Hendrix, J. W., Stuckey, R. E., and Bachi, P. R. 1990. Influence of plant date and cultivar on sudden death syndrome in Kentucky. Plant Dis. 74:761-766.

9. Hirrel, M. C. 1983. Sudden death syndrome of soybean: A disease of unknown etiology. Phytopathology 73:501-502.

10. Hooper, D. J. 1986. Extraction of free-living stages from soil. Pages 5-30 in: Laboratory Methods for Work with Plant and Soil Nematodes. 6th ed. J. F. Southey, ed. Ministry of Agriculture, Fisheries and Food, London.

11. Huang, Y. H., and Hartman, G. L. 1996. A semi-selective medium for detecting Fusarium solani, the causal organism of soybean sudden death syndrome. (Abstr.) Phytopathology 86(suppl.):S12. 
12. Jenkins, W. R. 1964. A rapid centrifugal-flotation technique for separating nematodes from soil. Plant Dis. Reptr. 48:692.

13. Jin, H., Hartman, G. L., Nickell, C. D., and Widholm, J. M. 1996. Phytotoxicity of culture filtrate from Fusarium solani, the causal agent of sudden death syndrome of soybean. Plant Dis. 80:922-927.

14. Jin, H., Hartman, G. L., Nickell, C. D., and Widholm, J. M. 1996. Characterization and purification of phytotoxin produced by Fusarium solani, the causal agent of sudden death syndrome of soybean. Phytopathology $86: 277-282$.

15. Khan, M. W. ed. 1993. Nematode Interactions. Chapman \& Hall, London.

16. Killebrew, J. F., Roy, K. W., and McLean, K. S. 1988. Greenhouse and field evaluation of Fusarium solani pathogenicity to soybean seedlings. Plant Dis. 72:1067-1070.

17. Köhl, J., and Gerlagh, M. 1999. Biological control of Botrytis cinerea in roses by the antagonist Ulocladium atrum. Mededelingen Faculteit Landbouwkundige en Toegepaste Biologische Wetenschappen Universityeit Gent. 64:441-445.

18. Lawrence, G. W., Roy, K. W., and McLean, K. S. 1988. Soybean cyst nematode associations with sudden death syndrome of soybeans. (Abstr.) Phytopathology 78:1514.

19. Malvick, D. K., and Grunden, E. 2005. Isolation of fungal DNA from plant tissues and removal of DNA amplification inhibitors. Mol. Ecol. Notes 5:958-960.

20. Martin, W. J., Newsom, L. D., and Jones, J. E. 1956. Relationship of nematodes to the development of Fusarium wilt in cotton. Phytopathology 46:285-289.

21. McLean, K. S., and Lawrence, G. W. 1993. Interrelationship of Heterodera glycines and Fusarium solani in sudden death syndrome of soybean. J. Nematol. 25:434-439.

22. McLean, K. S., and Lawrence, G. W. 1993. Localized influence of Heterodera glycines on sudden death syndrome of soybean. J. Nematol. 25:674-678.

23. McLean, K. S., and Lawrence, G. W. 1995. Development of Heterodera glycines as affected by Fusarium solani, the causal agent of sudden death syndrome of soybean. J. Nematol. 27:70-77.

24. McManus, P. S., Best, V. M., and Voland, R. P. 1999. Infection of cranberry flowers by Monilinia oxycocci and evaluation of cultivars for resistance to cottonball. Phytopathology 89:1127-1130.

25. Nash, S. M., and Snyder, W. C. 1962. Quantitative estimates by plate counts of propagules of the bean root rot Fusarium in field soils. Phytopathology 52:567-572.

26. Niblack, T. L., Arelli, P. R., Noel, G. R., Opperman, C. H., Orf, J. H., Schmitt, D. P., Shannon, J. G., and Tylka, G. L. 2002. A revised classification scheme for genetically diverse populations of Heterodera glycines. J. Nematol. 34:279-288.

27. Niblack, T. L., Heinz, R. D., Smith, G. S., and Donald, P. A. 1993. Distribution, density, and diversity of Heterodera glycines in Missouri. J. Nematol. 25:880-886.

28. Ortiz-Ribbing, L. M., and Eastburn, D. M. 2004. Soybean root systems and sudden death syndrome severity: Taproot and lateral root infection. Plant Dis. 88:1011-1016.

29. Roy, K. W., Hershman, D. E., Rupe, J. C., and Abney, T. S. 1997. Sudden death syndrome of soybean. Plant Dis. 81:1100-1111.

30. Roy, K. W., Lawrence, H. H., Hodges, H. H., McLean, K. S., and
Killebrew, J. F. 1989. Sudden death syndrome of soybean: Fusarium solani as incitant and relation of Heterodera glycines to disease severity. Phytopathology 79:191-197.

31. Rupe, J. C. 1997. Effect of crop rotation on soil population densities and Fusarium solani and Heterodera glycines and on the development of sudden death syndrome of soybean. Crop Prot. 16:575-580.

32. Rupe, J. C., Robbins, R. T., Becton, C. M., Sabbe, W. A., and Gbur, E. E., Jr. 1999. Vertical and temporal distribution of Heterodera glycines and Fusarium solani in fields with sudden death syndrome of soybean in two Arkansas fields. Soil Biol. Biochem. 31:245-251.

33. Rupe, J. C., Sabbe, W. E., Robbins, R. T., and Gbur, E. E., Jr. 1993. Soil and plant factors associated with sudden death syndrome of soybean. J. Prod. Agric. 6:218-221.

34. Russ, J. P. 1965. Predisposition of soybeans to Fusarium wilt by Heterodera glycines and Meloidogyne incognita. Phytopathology 55:361-364.

35. Scherm, H., Yang, X. B., and Lundeen, P. 1998. Soil variables associated with sudden death syndrome in soybean fields in Iowa. Plant Dis. 82:1152-1157.

36. Schmitt, D. P. 2004. Introduction. Pages 1-7 in: Biology and Management of Soybean Cyst Nematode. 2nd ed. D. P. Schmitt, J. A. Wrather, and R. D. Riggs, eds. Schmitt \& Associates, Marceline, MO.

37. Shaner, G., and Finney, R. E. 1977. The effect of nitrogen fertilization on the expression of slow-mildewing resistance in Knox wheat. Phytopathology 67:1051-1056.

38. Siddiqui, Z. K., Mir, R. A., and Mahmood, I. 1999. Effects of Meloidogyne incognita, Fusarium oxysporum f. sp. pisi, Rhizobium sp., and different soil types on growth, chlorophyll, and carotenoid pigments of pea. Israel J. Plant Sci. 47:251-256.

39. Sikora, R. A., and Carter, W. W. 1987. Nematode interactions with funga and bacterial plant pathogens: Fact or fantasy. Pages 307-312 in: Vistas on Nematology. J. A. Veech and D. W. Dickson, eds. Society of Nematologists, Hyattsville, MD.

40. Smith, G. S., and Carvil, O. N. 1997. Field screening of commercial and experimental soybean cultivars for their reaction to Macrophomina phaseolina. Plant Dis. 81:363-368.

41. Starr, J. L., Jeger, M. J., Martyn, R. D., and Schilling, K. 1989. Effects of Meloidogyne incognita and Fusarium oxysporum f. sp. vasinfectum on plant mortality and yield of cotton. Phytopathology 79:640-646.

42. Tabor, G. M., Tylka, G. L., Behm, J. E., and Bronson, C. R. 2003. Heterodera glycines infection increases incidence and severity of brown stem rot in soybean. Plant Dis. 87:655-661.

43. Wallace, H. R. 1978. The diagnosis of plant diseases of complex etiology. Annu. Rev. Phytopathol. 16:379-402.

44. Wang, J., Niblack, T. L., Tremain, J. A., Wiebold, W. J., Tylka, G. L., Marett, C. C., Noel, G. R., Myers, O., and Schmidt, M. E. 2003. The soybean cyst nematode reduces yield without causing obvious aboveground symptoms. Plant Dis. 87:623-628.

45. Webster, J. M. 1985. Interaction of Meloidogyne with fungi on crop plants. Pages 183-213 in: An Advanced Treatise on Meloidogyne. Vol. I: Biology and Control. J. N. Sasser and C. C. Carter, eds. North Carolina State University Graphics, Raleigh, NC

46. Wrather, J. A., Anderson, T. R., Arsyad, D. M., Cai, J., and Ploper, L. T. 1997. Soybean disease loss estimated for the top 10 soybean producing countries in 1994. Plant Dis. 81:107-110. 